\title{
Totally extraperitoneal inguinal hernia repair with or without fixation leads to similar results. Outcome of randomized prospective trial
}

\author{
Konrad Pielaciński ${ }^{1}$, Bartosz Puła르. Tadeusz Wróblewski ${ }^{3}$, Michał Kuryłowicz $^{1}$, Andrzej B. Szczepanik ${ }^{1}$ \\ ${ }^{1}$ Department of General, Oncologic and Metabolic Surgery, Institute of Hematology and Transfusion Medicine, Warsaw, Poland \\ ${ }^{2}$ Department of Hematology, Institute of Hematology and Transfusion Medicine, Warsaw, Poland \\ ${ }^{3}$ Department of General, Transplant and Liver Surgery, Medical University of Warsaw, Warsaw, Poland
}

Videosurgery Miniinv 2020; 15 (1): 1-10

DOI: https://doi.org/10.5114/wiitm.2019.83611

\begin{abstract}
Introduction: The use of implants in inguinal hernia repair has reduced its recurrence rate. However, postoperative groin pain still remains an unresolved problem. There are suggestions that in totally extraperitoneal inguinal hernia repair (TEP-IHR) two of the likely factors responsible for pain are use of fixation and the type of fixation used.

Aim: To evaluate the impact of mesh fixation on the incidence of postoperative pain, restriction of physical activities, hernia recurrence risk, return to normal activities and demand for analgesics in patients after unilateral TEP-IHR.

Material and methods: Unilateral TEP-IHR was performed in 139 male patients randomized to three groups: self-gripping mesh (SG), lightweight mesh (L) and lightweight mesh with fixation (LF). Full study-inclusion criteria were met by 110 patients; 43,18 and 49 in groups SG, L and LF respectively. Follow-up occurred on the $1^{\text {st }}, 2^{\text {nd }}$, and $7^{\text {th }}$ day and 3, 6, 12 months postoperatively. The numeric rating scale (NRS) was used to assess pain and the EuraHS-QOL (European Registry for Abdominal Wall Hernias Quality of Life Score) questionnaire to compare quality of life (QoL) prior to surgery and one year later.

Results: No statistically significant differences were observed between study groups with regard to the incidence rate and intensity of acute post-operative pain, chronic pain, analgesic demand, return to normal activity, hernia recurrence rate and post-operative QoL.

Conclusions: Lack of fixation in TEP-IHR does not increase the risk of hernia recurrence, and its presence does not significantly worsen the treatment results; especially it does not increase the incidence of chronic pain.
\end{abstract}

Key words: chronic pain, hernia recurrence, totally extraperitoneal inguinal hernia repair, mesh fixation.

\section{Introduction}

The use of synthetic meshes in inguinal hernia repair (IHR) has significantly reduced the groin hernia recurrence rate and markedly improved the treatment outcome. Nevertheless, postoperative pain still remains an unresolved clinical problem. Chronic postoperative pain of more than 3 months is estimated to occur in $2-53 \%$ of patients, and the occurrence rate primarily depends on the surgical technique. The chronic pain rate for laparoendoscopic techniques such as totally extraperitoneal approach (TEP) is estimated at approximately 6\% (range: 1-16\%), which is significantly lower than the rate for tension-free open hernia repair technique of approximately $18 \%$ (range: $1-75 \%$ ) [1-5]. The advantage of TEP-IHR is mainly due to less injury and

\section{Address for correspondence}

Konrad Pielaciński MD, Department of General, Oncologic and Metabolic Surgery, Institute of Hematology and Transfusion Medicine,

14 Indiry Gandhi St, 02-776 Warsaw, Poland, phone: +48 22 3496268, e-mail: konpiel@tlen.pl 
the dissection and implantation of the implant in the pre-peritoneal space. However, apart from the type of surgery technique, other factors that may affect the incidence of postoperative pain may be the type of implants used, the fixing of implants and the method of fixing them. In the case of implants, it is observed that without an increase of the risk of hernia recurrence, the use of a macroporous implant, called a light implant, is associated with a lower percentage of chronic pain than the use of socalled heavy implants. Fixation of the implant to the tissues is performed to prevent its movement and reduce the risk of recurrence. The use of traumatic methods for this purpose in the TEP-IHR, e.g. tacks, may prove to be a disadvantageous factor that increases the risk of damage to anatomical structures and the occurrence of chronic pain. Thus, the use of atraumatic alternatives such as self-gripping meshes or fibrin glue may contribute to lower postoperative pain [3, 6-12].

\section{Aim}

The study objective was to evaluate the impact of mesh fixation on the incidence of pain and pain-related restriction of physical activities, risk of hernia recurrence, return to preoperative activity and demand for analgesics in patients after TEP-IHR.

\section{Material and methods}

The randomized prospective clinical trial conducted between September 2012 and December 2016 included male patients of over 18 years of age scheduled for unilateral inguinal hernia repair, either primary or recurrent after open techniques. Patients after pelvic surgery from midline incisions below the umbilicus or with irreducible or incarcerated inguinal hernia or inguinoscrotal hernias were excluded from the study. Additionally, to limit the influence of other factors on the study aims from the final analysis, patients with adverse events such as hematomas in the groin or in the spermatic cord, wound infections or hernia recurrences were excluded. This study was approved by the local ethics committee (19/2011) and written informed consent was obtained from all the participants before the trial. Obligatory antibiotic prophylaxis was given at $2 \mathrm{~g}$ of cefazolin in a single dose and a $0.3 \mathrm{ml}$ s.c. dose of nadroparin was administered for prophylaxis of thromboembolic events. Random numbers were generated by the Randbetween function in the Microsoft Excel software, and patients were blinded and randomly divided into three groups. TEP-IHR was performed in the SG, L and LF groups using Parietex ProGrip mesh implants (Sofradim, Trevoux France), Ultrapro mesh (Ethicon, Diegem Belgium) or Ultrapro mesh and an AbsorbaTack Fixation Device (Covidien, Dublin Ireland) respectively. Patients were allowed to walk and drink after 4-6 h. Discharge occurred not earlier than $24 \mathrm{~h}$ after the operation and depended on the patient's general condition. Patients were instructed not to drive (for 7 days) or ride a bicycle (for 14 days) and warned against heavy lifting (for a month). A month following surgery no restrictions on physical activity were imposed. Paracetamol was prescribed to relieve pain. Data for the study were collected at the $1^{\text {st }}, 2^{\text {nd }}$, and $7^{\text {th }}$ (postoperative) day and 3, 6 and 12 months after the hernia repair procedure. Pain intensity was assessed on the NRS scale, where no pain corresponded to 0 , mild pain to $1-3$, moderate pain to $4-6$, and severe pain to $>6$ on the NRS. The EuraHS-QoL questionnaire was used to assess the intensity of groin pain and pain-related activity restrictions before surgery and 1 year after the procedure. The questionnaire is hernia-specific and includes 9 questions that can be scored by the patient on an 11-point scale from 0 to 10. The EuraHS-QoL questions are divided into 3 domains: "Pain" (range: 0-30), "Restriction of activities" (range: 0-40), and "Esthetic discomfort" (range: 0-20). The total score ranges from 0 to 90 , with the lower scores being the most favorable outcome. For many of the examined men, the esthetic/cosmetic aspect was of no importance so the "Esthetic discomfort" grades were dropped. As consequence, the EuraHS-QoL scale range was reduced from 0-90 to 0-70 [13].

\section{Surgical technique}

All TEP-IHR procedures were performed under general anaesthesia by one surgeon using a conventional three-port midline technique without the use of a balloon dissector [14]. In direct inguinal hernia procedures, the extended transversalis fascia was retracted and ligated with absorbable sutures. In indirect inguinal hernia procedures the hernial sac was completely dissected from the spermatic cord. Peritoneal lacerations enabling the intestines to enter the mesh (large tears) were closed with absorbable 
sutures, while remaining peritoneal defects were not sewn. Type of hernia was determined intraoperatively using the European Hernia Society (EHS) groin hernia classification. No drainage of the surgical site was performed [15].

\section{Mesh implantation}

A loosely rolled Parietex ProGrip mesh $(15 \times 9 \mathrm{~cm})$ with the gripping system on the outside was implanted through a $10 \mathrm{~mm}$ trocar. The gripping system (consisting of microgrips) was anchored to the tissues with a grasper. A rolled Ultrapro mesh $(15 \times 10 \mathrm{~cm})$ was inserted through a $10 \mathrm{~mm}$ port and arranged typically for the technique. The procedure for AbsorbaTacks Fixation Device implantation was as follows: the medial upper and lower mesh quadrants were fixed with two tacks each, the upper one to the rectus abdominis muscle and the lower one to the upper branch of the pubic bone. The upper lateral quadrant was fixed with one tack, while the lower one was left unfixed.

\section{Material characteristics}

The Parietex ProGrip implant is a large-pore polypropylene self-gripping mesh with polylactic acid microgrips resorbable after 15 months. Ultrapro mesh is a partially absorbable, macroporous implant that consists of monofilament polypropylene fibers and poliglecaprone-25 fibers resorbable after about 3 months. The AbsorbaTack Fixation Device contains screw-like synthetic fixation tacks. The tacks are $5 \mathrm{~mm}$ in size and their depth of tissue penetration is $4 \mathrm{~mm}$. The tacks are made of absorbable synthetic polyester copolymer derived from lactic and glycolic acid, poly(glycolide-co-L-lactide), and their absorption is essentially complete prior to 12 months.

\section{Statistical analysis}

The statistical analysis was performed using Prism 7.0 (GraphPad, La Jolla, CA, USA). The D'Agostino and Pearson normality test was used to assess the data distributions. The Mann-Whitney U-test and the Kruskal-Wallis test with post hoc Dunn's multiple comparison analysis were applied to compare the groups of data that did not meet the assumptions of the parametric test. The Wilcoxon matched-pairs signed rank test was used to analyze baseline results with those at particular follow-up time points. Fisher's exact test and the $\chi^{2}$ test were used to compare qualitative variables. Results were considered statistically significant when $p<0.05$ in all the analyses.

\section{Results}

One hundred thirty-nine patients were randomized to three study groups. They underwent elective TEP-IHR using the Parietex ProGrip mesh in the SG group (SG), Ultrapro mesh in the $L$ group $(\mathrm{L})$ and UItrapro mesh and the AbsorbaTack Fixation Device in the LF group (LF); 29 patients were excluded from the study - 9 (6.5\%) from SG, 8 (6.5\%) from L, and 12 (8.6\%) from LF. The reasons for exclusion were: groin and/or spermatic cord hematomas - 11 (8\%) patients (4 from SG, 1 from L, 6 from LF); bacterial infection of the surgical wound $-1(0.72 \%)$ patient from LF; exacerbation of comorbidities - 5 (3.6\%) patients ( 1 from SG, and 2 from $L$ and LF); failure to show up for follow-up visits - 10 (7.2\%) patients (3 from SG, 5 from L, 2 from LF). The frequency of adverse events did not differ significantly between the groups (Fisher's exact test). During the 1-year follow-up 2 hernia recurrences were reported, which accounts for $1.43 \%$ of all surgical patients. The first recurrence was reported 3 months after surgery in $P$, the second after 6 months in UA. The hernia recurrence rate in all study groups was statistically insignificant (Fisher's exact test). Prospective evaluation of treatment outcome was performed for 110 patients whose treatment course was uninterrupted (Figure 1). They were assigned to the following 3 groups: SG - 43 patients; L - 18 patients; and LF - 49 patients. Beside a slight statistical difference in the body mass index distribution among analyzed patients $(p=0.0426)$, no other statistically significant differences between groups with regard to patients' characteristics were found (Table I). Mean surgery time was $86.87 \pm 31.52 \mathrm{~min}$ and differed significantly between the groups ( $p=0.0085$, Kruskal-Wallis test). The post-host analysis showed significant increase in overall procedure surgery time for patients in the SG group ( $p<0.01$, Dunn's multiple comparison analysis) as compared to patients in the LF group. Peritoneal lacerations during surgery occurred in $54(49.1 \%)$ patients, mostly in the LF group - 31 (63.3\%), less often in the SG group - 16 $(32.7 \%)$ and 7 (38.9\%) in the $L$ group. The differences were statistically significant ( $p<0.05 ; \chi^{2}$ test). In 24 cases the lacerations were large (large tears) and 


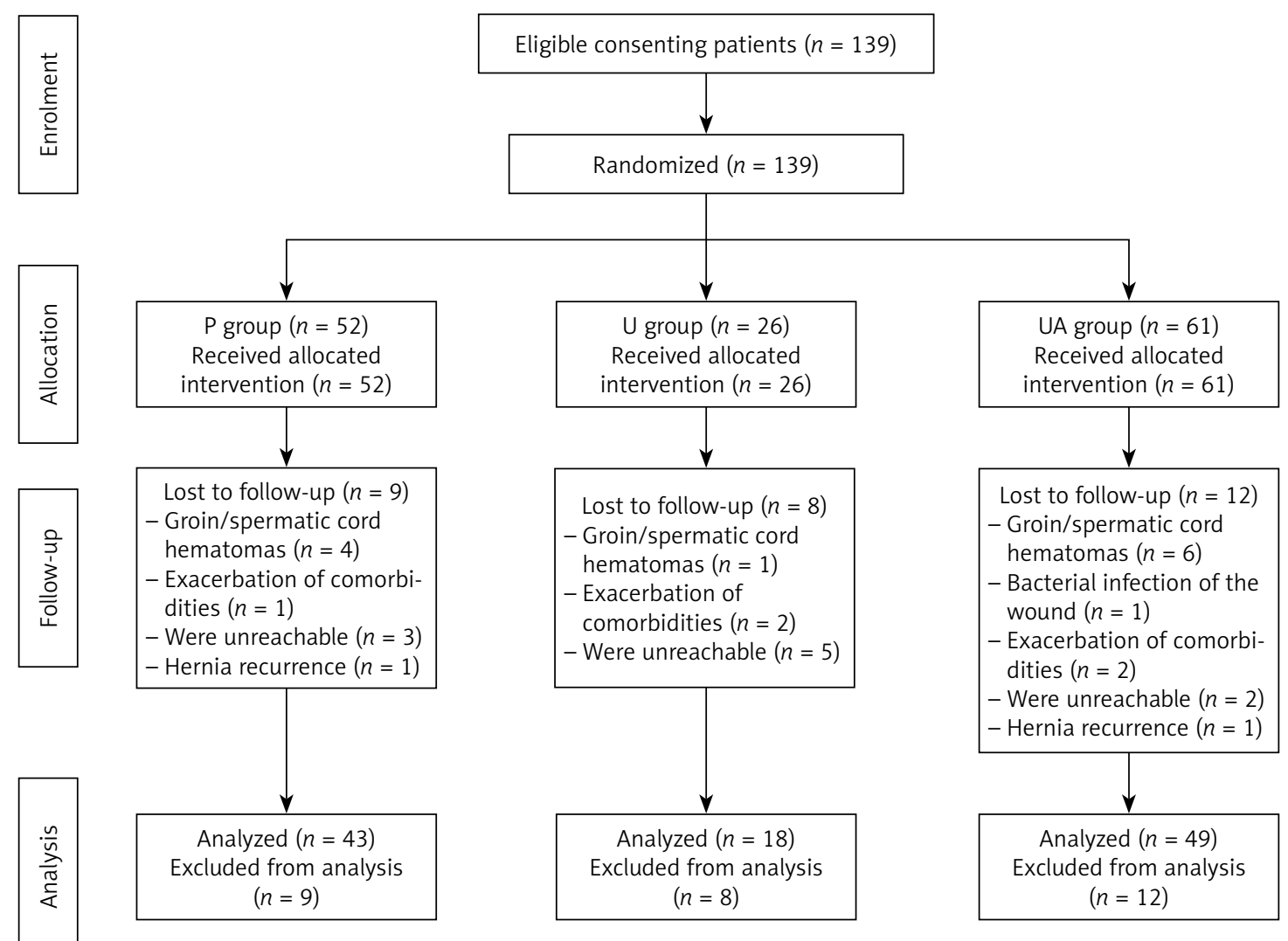

Figure 1. Study flow chart

required stitches, while in 30 cases no stitches were necessary. Peritoneal lacerations were observed to significantly prolong surgery time $(77 \pm 28$ min vs. 96 \pm 31 min, $p<0.005$ Mann-Whitney test). On average, discharge took place after $2.67 \pm 2.07$ days in group SG; $2.56 \pm 1.82$ days in $L$ and $2.59 \pm 2.11$ days in LF. No statistically significant differences were observed with regard to hospital stay (Dunn's multiple comparison test). Return to pre-operative activity measured in days did not differ significantly between the groups (Dunn's multiple comparison test), although it was the shortest for patients in the L group, at 2.72 \pm 1.18 days, whereas in the SG and LF groups it was $3.16 \pm 0.99$ days and $3.082 \pm 1.08$ days respectively. Before surgery 97 (88.2\%) patients reported groin pain: 39 from SG, 16 from $L$ and 42 from LF. Pain was assessed as mild by 39 (35.5\%) patients (14 - SG, $5-\mathrm{L}, 20-\mathrm{LF}$ ), moderate by 33 (30\%) patients (16SG, 9 - L, 8 - LF) and severe by 25 (22.7\%) patients (9-SG, 2 - L, 14 - LF). Only patients with moderate to severe pain (> 4 according to NRS) used painkillers on an ad-hoc basis, and pain intensity did not differ significantly between groups in the pre-surgery period ( $\chi^{2}$ test). No statistically significant differences between groups were observed with regard to pain intensity in the early post-operative period as assessed on the $1^{\text {st }}, 2^{\text {nd }}$ and $7^{\text {th }}$ days ( $\chi^{2}$ test). On the $8^{\text {th }}$ post-surgery day a statistically significant reduction in pain intensity was reported as compared to the $1^{\text {st }}$ and $2^{\text {nd }}$ days, though no differences between groups were observed ( $p<0.005$ in SG and L group and $p<0.0001$ in LF group, Dunn's multiple comparison test). Average demand for painkillers calculated until day $7^{\text {th }}$ was as follows: $0.95 \pm 9.48 \mathrm{~g}$ in group SG, $0.96 \pm 0.92 \mathrm{~g}$ in group $\mathrm{L}$ and $1.02 \pm 1.10 \mathrm{~g}$ in group LF. There were no statistically significant differences with regard to the implant used (Dunn's multiple comparison test) (Table II). Three months after surgery, 63 (57.3\%) patients, including 26 in group SG, 9 in group L and 28 in group LF group reported chronic pain in the groin. The pain was assessed as mild by 55 (50\%) patients ( 22 - SG, $8-L, 25-L F)$, moderate by 7 (6.3\%) patients (4 - SG, 1 - L, 2 - LF) and severe by 1 (1\%) patient from the LF group. Six 
Table I. Demographic and surgery-related parameters

\begin{tabular}{|c|c|c|c|c|}
\hline Parameter & SG group $(n=43)$ & L group $(n=18)$ & LF group $(n=49)$ & $P$-value \\
\hline Age, mean \pm SD [years] & $49.3 \pm 14.9$ & $47.3 \pm 10.4$ & $50.1 \pm 13.6$ & $0.7365^{b}$ \\
\hline BMI: & & & & $0.0426^{a}$ \\
\hline Normal & $26(60.5)$ & $13(72.2)$ & $22(44.9)$ & \\
\hline Overweight & $15(34.9)$ & $5(27.8)$ & $17(34.7)$ & \\
\hline Obese & $2(4.6)$ & $0(0.0)$ & $10(20.4)$ & \\
\hline Previous surgery: & & & & $0.9736^{a}$ \\
\hline Yes & $23(53.5)$ & $10(55.5)$ & $28(57.1)$ & \\
\hline No & $20(46.5)$ & $8(44.4)$ & $21(42.8)$ & \\
\hline Comorbidities: & & & & $0.3854^{a}$ \\
\hline Yes & $23(53.5)$ & $9(50.0)$ & $32(65.3)$ & \\
\hline No & $20(46.5)$ & $9(50.0)$ & $17(34.7)$ & \\
\hline ASA class: & & & & $0.1803^{a}$ \\
\hline 1 & $20(46.5)$ & $9(50.0)$ & $17(34.7)$ & \\
\hline$\|$ & $20(46.5)$ & $6(33.3)$ & $30(61.2)$ & \\
\hline III & $3(7.0)$ & $3(16.6)$ & $2(4.1)$ & \\
\hline Hernia side: & & & & $0.0803^{a}$ \\
\hline Right-sided & $29(67.4)$ & $7(38.9)$ & $23(46.9)$ & \\
\hline Left-sided & $14(32.6)$ & $11(61.1)$ & $26(53.1)$ & \\
\hline \multicolumn{5}{|c|}{ Hernia type - EHS classification: } \\
\hline Lateral: & & & & $0.1707^{\mathrm{a}}$ \\
\hline । & $9(20.9)$ & $3(16.6)$ & $5(10.2)$ & \\
\hline$\|$ & $5(11.6)$ & $5(27.8)$ & $2(4.1)$ & \\
\hline III & $15(34.9)$ & $5(27.8)$ & $16(32.6)$ & \\
\hline Medial: & & & & $0.2000^{\mathrm{a}}$ \\
\hline I & $2(4.6)$ & $2(11.1)$ & $4(8.1)$ & \\
\hline$\|$ & $7(16.3)$ & $1(5.5)$ & $5(10.2)$ & \\
\hline III & $5(11.6)$ & $2(11.1)$ & $16(32.6)$ & \\
\hline Incarceration: & & & & $0.5651^{\mathrm{a}}$ \\
\hline Yes & $5(11.6)$ & $4(22.2)$ & $8(16.3)$ & \\
\hline No & $38(88.4)$ & $14(77.7)$ & $41(83.7)$ & \\
\hline Other hernias: & & & & $0.5707^{a}$ \\
\hline Yes & $5(11.6)$ & $1(5.5)$ & $3(6.1)$ & \\
\hline No & 38 (88.4) & $17(94.4)$ & $46(93.9)$ & \\
\hline
\end{tabular}

Data are presented as numbers of patients (n) and percentages (\%) or mean $\pm S D .{ }^{a}$ Calculated with $\chi^{2}$ test; ${ }^{b}$ compared using Kruskal-Wallis test. EHS - European Hernia Society, ASA - American Society of Anesthesiologists, BMI - body mass index, SD - standard deviation. 
Table II. Overall comparison of surgery-related and outcome parameters

\begin{tabular}{|c|c|c|c|c|}
\hline Parameter & $\begin{array}{l}\text { SG group } \\
(n=43)\end{array}$ & $\begin{array}{l}\text { L group } \\
(n=18)\end{array}$ & $\begin{array}{l}\text { LF group } \\
(n=49)\end{array}$ & $P$-value \\
\hline Duration of operation, mean $\pm \mathrm{SD}[\mathrm{min}]$ & $76.7 \pm 26.8$ & $80.2 \pm 19.0$ & $98.3 \pm 35.5$ & $0.0085^{b}$ \\
\hline Peritoneal tear: & & & & $0.0285^{a}$ \\
\hline Yes & $16(37.2)$ & $7(38.9)$ & $31(63.3)$ & \\
\hline No & $27(62.8)$ & $11(60.1)$ & $18(36.7)$ & \\
\hline $\begin{array}{l}\text { Analgesic consumption on } 1^{\text {st }}, 2^{\text {nd }} \\
\text { and } 7^{\text {th }} \text { postoperative days, mean } \pm S D[g]\end{array}$ & $0.95 \pm 9.48$ & $0.96 \pm 0.92$ & $1.02 \pm 1.10$ & $0.9916^{b}$ \\
\hline Length of postoperative stay, mean \pm SD [days] & $2.67 \pm 2.1$ & $2.56 \pm 1.8$ & $2.59 \pm 2.11$ & $0.9670^{b}$ \\
\hline Return to pre-operative activity, mean \pm SD [days] & $3.16 \pm 0.99$ & $2.72 \pm 1.18$ & $3.082 \pm 1.08$ & $0.3063^{a}$ \\
\hline
\end{tabular}

Data are presented as numbers of patients ( $n$ ) and percentages (\%) or mean $\pm S D .{ }^{a}$ Calculated with $\chi^{2}$ test; ${ }^{b}$ compared using Kruskal-Wallis test.

months following surgery chronic groin pain was reported by 33 (30\%) patients, including 13 patients in group SG, 2 in group L and 16 in group LF. The pain was assessed as mild by $29(24.4 \%)$ patients (11SG, $2-L, 16-L F)$, and moderate by 4 (3.6\%) patients (two in each group SG and LF). No severe pain was reported. Twelve months after surgery chronic groin pain was reported by 23 (20.9\%) patients, including 8 in group SG, 1 in group L and 14 in group LF. The pain was assessed as mild by $22(20 \%)$ patients (8-SG, $1-L, 13-L F)$, and moderate by 1 (1\%) patient from the LF group. No severe pain was reported. No statistically significant differences between the study groups were found ( $\chi^{2}$ test) with regard to the incidence rate and intensity of postoperative chronic groin pain at 3, 6 and after 12 months following surgery (Table III). Analgesic treatment was limited to ad-hoc ingestion of pain killers. Post-operative pain assessment revealed a time-related decrease in the number of patients suffering pain as well as reduction of pain intensity $(p<0.0001$; Kruskal-Wallis test), but no significant differences between analyzed groups. Average pain intensity and pain-related movement restrictions were assessed using the EuraHS-QoL questionnaire prior to surgery and one year after the procedure. Comparison of results from before surgery and 1 year after surgery in the SG, L, LF study groups (Dunn's multiple comparison test) revealed no statistically significant differences in either domain (Table IV). However, comparison of results for all patients from before surgery and one year later revealed a statistically significant decrease of pain intensity $(p<0.0001$ for all analyses, Wilcoxon matched-pairs signed rank test) from 7.62 to
0.85 as well as pain-related activity restrictions from 14.8 to 0.77 (Table V).

\section{Discussion}

Implants in hernia repair have greatly contributed to improvement of treatment effects, particularly to a marked decrease in hernia recurrence rate. Nevertheless, post-operative groin pain still remains an unresolved clinical problem, even if minimally invasive techniques are used (e.g. laparoendoscopic $\mathrm{IH}$ repair). Each surgical technique has its own typical pain incidence rate, and pain intensity sometimes restricts the patients' physical activity. This incidence for TEP techniques ranges from $1 \%$ to $16 \%$ and it is reported in $6-8 \%$ of patients on average $[1-4,6-8,16,17]$. In our prospective randomized trial this percentage was slightly higher and one year following surgery chronic pain was reported in 23 (20.9\%) patients, although 22 of them reported mild pain and only slight physical activity restriction (0.77 for restriction domain on the EuraHS-QoL questionnaire). Moreover, a year after surgery (TEP-IHR) we observed a favorable tendency of pain regression or significant decrease in chronic pain intensity. This has been confirmed in similar publications $[9,17$, 18]. On the other hand, the surgical outcome in the study groups (P, $U$ and $U A$ ) did not significantly differ with regard to incidence rate and intensity of chronic pain as assessed after 3, 6 and 12 months or pain decrease or regression a year after surgery. Thus, our results do not confirm the adverse effect of fixation on the risk of chronic pain, which is in accordance with results of similar studies and meta-analyses $[6,16-20]$. On the other hand, the outcome of our 
Table III. Patient-reported pain using NRS scale prior to surgery and at follow-up evaluation. 0 - no pain, 1-3 - mild pain, 4-6 - moderate pain, > 6 - severe pain

\begin{tabular}{|c|c|c|c|c|}
\hline Pain & SG group $(n=43)$ & L group $(n=18)$ & LF group $(n=49)$ & $P$-value \\
\hline Before surgery: & & & & $0.1547^{a}$ \\
\hline No pain & 4 & 2 & 7 & \\
\hline Mild pain & 14 & 5 & 20 & \\
\hline Moderate pain & 16 & 9 & 8 & \\
\hline Severe pain & 9 & 2 & 14 & \\
\hline $1^{\text {st }}$ day: & & & & $0.3091^{a}$ \\
\hline No pain & 2 & 0 & 0 & \\
\hline Mild pain & 17 & 11 & 24 & \\
\hline Moderate pain & 14 & 4 & 19 & \\
\hline Severe pain & 10 & 3 & 6 & \\
\hline $2^{\text {nd }}$ day: & & & & $0.3391^{a}$ \\
\hline No pain & 2 & 2 & 0 & \\
\hline Mild pain & 22 & 8 & 32 & \\
\hline Moderate pain & 13 & 5 & 12 & \\
\hline Severe pain & 6 & 3 & 5 & \\
\hline $7^{\text {th }}$ day: & & & & $0.4025^{a}$ \\
\hline No pain & 2 & 1 & 5 & \\
\hline Mild pain & 33 & 11 & 38 & \\
\hline Moderate pain & 6 & 5 & 6 & \\
\hline Severe pain & 2 & 1 & 0 & \\
\hline 3 months: & & & & $0.8447^{\mathrm{a}}$ \\
\hline No pain & 17 & 9 & 21 & \\
\hline Mild pain & 22 & 8 & 25 & \\
\hline Moderate pain & 4 & 1 & 2 & \\
\hline Severe pain & 0 & 0 & 1 & \\
\hline 6 months: & & & & NA \\
\hline No pain & 30 & 16 & 31 & \\
\hline Mild pain & 11 & 2 & 16 & \\
\hline Moderate pain & 2 & 0 & 2 & \\
\hline Severe pain & 0 & 0 & 0 & \\
\hline 12 months: & & & & NA \\
\hline No pain & 35 & 17 & 35 & \\
\hline Mild pain & 8 & 1 & 13 & \\
\hline Moderate pain & 0 & 0 & 1 & \\
\hline Severe pain & 0 & 0 & 0 & \\
\hline
\end{tabular}

Data are presented as numbers of patients (n). ${ }^{a}$ Calculated with $\chi^{2}$ test; $N A-$ not available. 
Table IV. Outcome of measurement of EuraHS-QoL score of patients in the groups

\begin{tabular}{|c|c|c|c|c|}
\hline EuraHS-QoL score & $\begin{array}{l}\text { SG group } \\
(n=43)\end{array}$ & $\begin{array}{l}\text { L group } \\
(n=18)\end{array}$ & $\begin{array}{l}\text { LF group } \\
(n=49)\end{array}$ & $P$-value \\
\hline \multicolumn{5}{|l|}{ Preoperative: } \\
\hline Pain domain, mean (range) & $7.33(0-16)$ & $7.22(1-15)$ & $8.04(0-20)$ & $0.8418^{b}$ \\
\hline Restrictions domain, mean (range) & $14.12(0-34)$ & $12.61(0-30)$ & $16.29(0-38)$ & $0.2780^{b}$ \\
\hline \multicolumn{5}{|l|}{12 months after operation: } \\
\hline Pain domain, mean (range) & $0.79(0-8)$ & $0.38(0-3)$ & $1.08(0-7)$ & 0.0694 \\
\hline Restrictions domain, mean (range) & $0.69(0-15)$ & $0.41(0-2)$ & $1.08(0-12)$ & $0.0598^{t}$ \\
\hline
\end{tabular}

${ }^{b}$ Compared using Kruskal-Wallis test; EuraHS-QoL score - max value 70; pain domain, max. value 30; restrictions domain max. value 40.

Table V. Outcome of measurement of EuraHS-QoL score of patients - evaluation of TEP-IHR effectiveness

\begin{tabular}{|c|c|c|c|}
\hline EuraHS-QoL score & Preoperative & $\begin{array}{c}12 \text { months after } \\
\text { operation }\end{array}$ & $P$-value \\
\hline Pain domain, mean (range); all patients: & $7.62(1-20)$ & $0.85(0-8)$ & $<0.0001^{c}$ \\
\hline \multicolumn{4}{|l|}{ Pain domain, mean (range); in groups: } \\
\hline SG group & $7.33(0-16)$ & $0.79(0-8)$ & $<0.0001^{c}$ \\
\hline L group & $7.22(1-15)$ & $0.38(0-3)$ & $<0.0001^{c}$ \\
\hline LF group & $8.04(0-20)$ & $1.08(0-7)$ & $<0.0001^{c}$ \\
\hline Restrictions domain, mean (range); all patients: & $14.8(0-38)$ & $0.77(0-15)$ & $<0.0001^{c}$ \\
\hline \multicolumn{4}{|l|}{ Restrictions domain, mean (range); in groups: } \\
\hline SG group & $14.12(0-34)$ & $0.69(0-15)$ & $<0.0001^{c}$ \\
\hline L group & $12.61(0-30)$ & $0.41(0-2)$ & $<0.0001^{c}$ \\
\hline LF group & $16.29(0-38)$ & $1.08(0-12)$ & $<0.0001^{c}$ \\
\hline
\end{tabular}

cWilcoxon matched-pairs test; EuraHS-QoL score-max value 70; pain domain max. value 30; restrictions domain max. value 40.

study is contradictory to the study results of some other authors [9-11, 21]. During 1 year follow-up we found no statistically significant differences between study groups with regard to intensity of acute pain or demand for pain killers in the early post-operative period and later. Our results are similar to those of other studies $[7,8,18]$, but there are also publications that report divergent observations, i.e. after mesh fixation pain is more intense [15-17, 21]. Although the differences in return to normal activity were statistically insignificant $(\mathrm{L}-2.72 \pm 1.18$ days, SG $-3.16 \pm 0.99$, LF $-3.082 \pm 1.08)$ the values were slightly higher than reported in similar studies (especially for ProGrip implant - 1.6 days) but comparable to results of the published meta-analyses [19, 21-23]. Earlier publications on laparoendoscopic IH repair support the idea that hernia recurrence can be prevented by using suitable size mesh with fixation
$[21,24,25]$. With time and experience however the need for mesh fixation became a widely discussed problem mostly because of higher costs, more injury to tissue, greater risk of damage to anatomical structures, incidence of chronic pain as well as a limited impact on hernia recurrence. Despite such observations, fixation is still used for fear of hernia recurrence $[7,8]$. Numerous later reports confirmed that most patients do not require mesh fixation as the intra-abdominal pressure evenly presses the implant placed extraperitoneally on the posterior abdominal wall to the surrounding tissues, which prevents mesh displacement and hernia recurrence. Such immobilization plays a crucial role in mesh stabilization during the first 2 postoperative months, and within the first 2 postoperative weeks migration of immune cells and fibroblasts is observed, followed by collagen deposition leading to in-growth and per- 
manent mesh stabilization [24-28]. It is currently believed that hernia recurrence following the TEP technique is mostly due to insufficient tissue overlap; the surface of the mesh does not properly cover the hernia orifice so intra-abdominal pressure is not sufficient for mesh stabilization, which may lead to mesh dislocation and hernia recurrence. It is therefore recommended to perform an adequately wide exposure of the myopectineal orifice to allow the use of a large $15 \times 10$ or $15 \times 15 \mathrm{~cm}$ mesh [5, 26-28]. If sufficient tissue overlap is impossible, e.g., during medial hernias with a defect size larger than $3 \mathrm{~cm}$, it is advisable to use additional implant fixation [5, 16]. In our study, flat mesh and issues related to its fixation were evaluated. However, the next factor that may influence the results, including the risk of recurrence or the frequency of postoperative pain, may be the shape of an implant. So-called three-dimensional (3D) mesh, available on the market, has a shape adapted to the anatomy of the groin, and its design minimizes buckling and reduces the need for fixation. Therefore, it is believed to be easier to use, thus reducing the time of surgery. On the other hand, the anatomical shape better fits the tissues and minimizes the need for mesh fixation [11]. In our study we report two hernia recurrences $(1.43 \%)$ which is an acceptable rate comparable to that reported in other literature data [18], although there are also reports of no recurrences whatsoever [21, 27]. The first hernia recurrence occurred 3 months after surgery in the SG group, the other after 6 months in the LF group. Although there were no statistically significant differences between our study groups with regard to recurrence rate (1 - SG, $0-L, 1-L F)$, as in other reports $[19,29]$, the result is noteworthy because the two cases were reported for patients with $L 1$ and $L 2$ hernias operated on with mesh fixation (with either microgrips of implant or tacks), which was supposed to limit the risk of hernia recurrence [21, 24, 25]. During surgery L2 hernias were detected and resolved with the Lichtenstein technique. Most likely the hernia recurrence was due to surgical error related to inadequate separation of the peritoneum and insufficient overlap. Less likely are surgical errors related to mesh dislocation during desufflation, which usually manifest immediately after surgery. We are aware that our study groups are relatively small and our results need confirmation in larger cohorts, but we did not observe any marked unfavorable effect of implant fixation or benefits from the use of the Parietex ProGrip self-fixing mesh. Our observations regarding the ProGrip self-fixing mesh are not confirmed in the studies of other authors, who demonstrated its beneficial treatment effect mostly reflected in the reduction of post-operative pain, faster return to normal physical activity or lack of hernia recurrence $[11,22,23,30,31]$. Most of the studies have shown that non-fixation hernia repair is generally more cost-effective than repair with mesh fixation. Cost of TEP-IHR and feasibility of TEP-IHR with ProGrip were not evaluated in this study. Nevertheless, the use of self-gripping mesh eliminates the expense of a fixation device, therefore decreasing the overall cost of the TEP-IHR. However, in our opinion, the use of the ProGrip implant is technically more difficult and requires a longer operation time than using a light mesh with or without fixation. In our study, comparison of pre-surgery and post-surgery quality of life based on the EuraHS-QoL questionnaire indicates high treatment efficacy. Regardless of the type of implant or mesh fixation technique, TEP-IHR significantly contributes to improvement of the patients' quality of life. With hernia removal the pain subsides or is markedly reduced, as is pain-related physical restriction. Our results are confirmed in the reports of other authors where TEP-IHR is also given a high rating $[11,13,16,17]$.

\section{Conclusions}

The TEP-IHR with implants and fixation techniques described in our study is effective for prevention of hernia recurrence. Additional mesh fixation does not increase the risk of post-operative chronic pain, and the pain following the procedure is usually mild and does not significantly restrict physical activity or impair the quality of life.

\section{Conflict of interest}

The authors declare no conflict of interest.

\section{References}

1. Eklund A, Montgomery A, Bergkvist L, Rudberg C; the Swedish Multicentre Trial of Inguinal Hernia Repair by Laparoscopy (SMIL) study group. Chronic pain 5 years after randomized comparison of laparoscopic and Lichtenstein inguinal hernia repair. Br J Surg 2010; 97: 600-8.

2. Reinpold W. Risk factors of chronic pain after inguinal hernia repair: a systematic review. Innov Surg Sci 2017; 2: 61-8. 
3. McCormack K, Scott NW, Go PM, et al.; EU Hernia Trialists. Collaboration. Laparoscopic techniques versus open techniques for inguinal hernia repair. Cochrane Database Syst Rev 2003; 1: CD001785.

4. Mitura K, Dąbrowiecki S, Śmietański M, Matyja A. The experience and awareness of laparoendoscopic procedures among Polish surgeons in everyday clinical practice. Videosurgery Miniinv 2017; 12: 13-8.

5. The HerniaSurge Group. International guidelines for groin hernia management. Hernia 2018; 22: 1-165.

6. Mitura K, Garnysz K, Wyrzykowska D, Michałek I. The change in groin pain perception after transabdominal preperitoneal inguinal hernia repair with glue fixation: a prospective trial of a single surgeon's experience. Surg Endosc 2018; 32: 4284-9.

7. Tam KW, Liang HH, Chai CY. Outcomes of staple fixation of mesh versus nonfixation in laparoscopic total extraperitoneal inguinal repair: a meta-analysis of randomized controlled trials. World J Surg 2010; 34: 3065-74.

8. Teng YJ, Pan SM, Liu YL, et al. A meta-analysis of randomized controlled trials of fixation versus nonfixation of mesh in laparoscopic total extraperitoneal inguinal hernia repair. Surg Endosc 2011; 25: 2849-58.

9. Fortelny RH, Petter-Puchner AH, Glaser KS, Redl H. Use of fibrin sealant (Tisseel/Tissucol) in hernia repair: a systematic review. Surg Endosc 2012; 26: 1803-12.

10. Chan MS, Teoh AY, Chan KW, et al. Randomized double-blinded prospective trial of fibrin sealant spray versus mechanical stapling in laparoscopic total extraperitoneal hernioplasty. Ann Surg 2014; 259: 432-7.

11. Yildirim D, Hut A, Uzman S. Spinal anesthesia is safe in laparoscopic total extraperitoneal inguinal hernia repair. A retrospective clinical trial. Videosurgery Miniinv 2017; 12: 417-27.

12. Bresnahan E, Bates A, Wu A, et al. The use of self-gripping (Progrip $^{\mathrm{TM}}$ ) mesh during laparoscopic total extraperitoneal (TEP) inguinal hernia repair: a prospective feasibility and long-term outcomes study. Surg Endosc 2015; 29: 2690-6.

13. Muysoms FE, Vanlander A, Ceulemans R, et al. A prospective, multicenter, observational study on quality of life after laparoscopic inguinal hernia repair with ProGrip laparoscopic, self-fixating mesh according to the European Registry for Abdominal Wall Hernias Quality of Life Instrument. Surgery 2016; 160: 1344-57.

14. Putnis S, Berney CR. Totally extraperitoneal repair of inguinal hernia: techniques and pitfalls of a challenging procedure. Langenbecks Arch Surg 2012; 397: 1343-51.

15. Miserez M, Alexandre JH, Campanelli G, et al. The European Hernia Society groin hernia classification: simple and easy to remember. Hernia 2007; 11: 113-6.

16. Bittner R, Montgomery MA, Arregui E, et al. Update of guidelines on laparoscopic (TAPP) and endoscopic (TEP) treatment of inguinal hernia (International Endohernia Society) [International Endohernia Society (IEHS)]. Surg Endosc 2015; 29: 289-321.

17. Burgmans JP, Schouten N, Clevers GJ, et al. Pain after totally extraperitoneal (TEP) hernia repair might fade out within a year. Hernia 2015; 19: 579-85.
18. Gutlic N, Rogmark P, Nordin P, et al. Impact of mesh fixation on chronic pain in total extraperitoneal inguinal hernia repair (TEP): a nationwide register-based study. Ann Surg 2016; 263 : 1199-206.

19. Buyukasik K, Ari A, Akce B, et al. Comparison of mesh fixation and non-fixation in laparoscopic totally extraperitoneal inguinal hernia repair. Hernia 2017; 21: 543-8.

20. Sajid MS, Ladwa N, Kalra L, et al. A meta-analysis examining the use of tacker fixation versus no-fixation of mesh in laparoscopic inguinal hernia repair. Int J Surg 2012; 10: 224-31.

21. Taylor C, Layani L, Liew V, et al. Laparoscopic inguinal hernia repair without mesh fixation, early results of a large randomised clinical trial. Surg Endosc 2008; 22: 757-62.

22. Bresnahan E, Bates A, Wu A, et al. The use of self-gripping (Progrip $^{\mathrm{TM}}$ ) mesh during laparoscopic total extraperitoneal (TEP) inguinal hernia repair: a prospective feasibility and long-term outcomes study. Surg Endosc 2015; 29: 2690-6.

23. Tolver MA, Rosenberg J, Bisgaard T. Convalescence after laparoscopic inguinal hernia repair: a qualitative systematic review. Surg Endosc 2016; 30: 5165-72.

24. Schwab R, Schumacher O, Junge K, et al. Biomechanical analyses of mesh fixation in TAPP and TEP hernia repair. Surg Endosc 2008; 22: 731-8.

25. Chowbey PK, Bandyopadhyay SK, Sharma A, et al. Recurrent hernia following endoscopic total extraperitoneal repair. J Laparoendosc Adv Surg Tech 2003; 13: 21-5.

26. Choy C, Shapiro K, Patel S, et al. Investigating a possible cause of mesh migration during totally extraperitoneal (TEP) repair. Surg Endosc 2004; 18: 523-5.

27. Garg P, Nair S, Shereef M, et al. Mesh fixation compared to nonfixation in total extraperitoneal inguinal hernia repair: a randomized controlled trial in a rurl center in India. Surg Endosc 2011; 25: 3300-6.

28. Claus CMP, Rocha GM, Campos ACL, et al. Mesh displacement after bilateral inguinal hernia repair with no fixation. JSLS 2017; 21: e2017.00033.

29. Ismail M, Garg P. Laparoscopic inguinal total extraperitoneal hernia repair under spinal anesthesia without mesh fixation in 1220 hernia repairs. Hernia 2009; 13: 115-9.

30. Birk D, Hess S, Garcia-Pardo C. Low recurrence rate and low chronic pain associated with inguinal hernia repair by laparoscopic placement of Parietex ProGrip ${ }^{\mathrm{TM}}$ mesh: clinical outcomes of 220 hernias with mean follow-up at 23 months. Hernia 2013; 17: 313-20.

31. Yildirim D, Donmez T, Ozcevik H, et al. Comparison of slit mesh versus nonslit mesh in laparoscopic extraperitoneal hernia repair. Videosurgery Miniinv 2018; 13: 469-76.

Received: 19.12.2018, accepted: 3.02.2019. 